Infos zu pflanzlicher

Halsschmerztablette

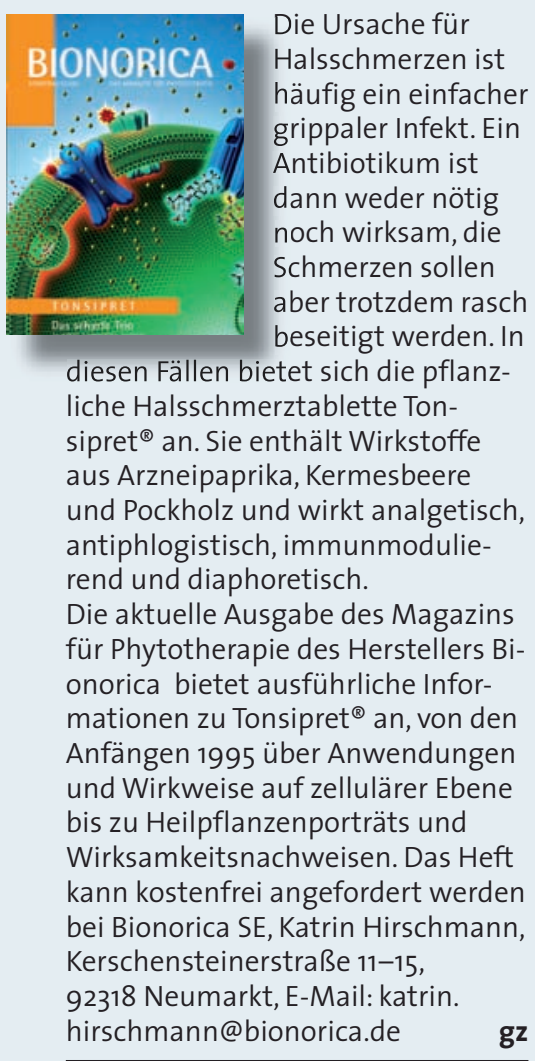

Nach Informationen von Bionorica

\section{Verbesserte Nachsorge}

durch IT

Ein Beispiel erfolgreicher IT-Nutzung präsentierte Dipl.-Ing Mark Winter, stellvertretender Geschäftsführer von auric ${ }^{\circledR}$ Hörsysteme, auf der Veranstaltung „eHealth.Niedersachsen Fokus Hannover": Telemedizin in der Nachsorge von Patienten mit einem Cochlea-Implantat. Damit die Patienten, denen in der Medizinischen Hochschule Hannover ein Cochlea-Implantat eingesetzt wurde, für die lebenslang notwendige jährliche Nachsorge keine weite Reise in Kauf nehmen müssen, hat auric ${ }^{\circledR}$ Hörsysteme Remote-Fitting entwickelt. Dank dieses Systems können Patienten die Nachsorge in einem der über 20 bundesweiten auric Hör- und Tinnituszentren durchführen lassen. Diese sind per Datenleitung mit der $\mathrm{MHH}$ verbunden.

Nach Informationen von auric ${ }^{\circledR}$ Hörsysteme

\title{
Kopf-Hals-Tumore: Erstmals Fortschritte durch zielgerichtete Therapie
}

\begin{abstract}
- Bei rezidivierten und/oder metastasierten Kopf-Hals Tumoren (SCCHN, squamous cell carcinoma of the head and neck) war das Patienten-Outcome in den letzten 30 Jahren frustran. Trotz neuer Substanzen und Kombinationen stagnierte das mediane Überleben bei einem halben Jahr und das 1-Jahres-Überleben bei 20-40\%. Erst die zielgerichtete Therapie mit Cetuximab (Erbitux $\left.{ }^{\circledR}\right)$ - zusätzlich zur Chemotherapie verabreicht - änderte die Situation: Mit dem monoklonalen Antikörper gegen den epidermalen Wachstumsfaktorrezeptor (EGFR, epidermal growth factor receptor) konnte erstmals das Gesamtüberleben und das progressionsfreie Überleben signifikant verlängert werden, wie Dr. Bert Hildebrandt aus Berlin berichtete. Das zeigte die EXTREMEStudie [Vermorken JB et al., NEJM 2008; 359: 1116-27], die 2008 zur Zulassung des EGFRAntikörpers in dieser Indikation führte. In der multizentrischen randomisierten Phase III-Studie erhielten 442 nicht vorbehandelte Patienten mit rezidiviertem und/oder metastasiertem SCCHN Cetuximab zusätzlich zur einer platinbasierten Chemotherapie (Cisplatin oder Carboplatin) und 5-Fluorouracil (5-FU) oder Chemotherapie allein. Verabreicht wurden maximal sechs Zyklen, an die sich im Prüfarm eine Erhaltung mit Cetuximab bis zum Progress anschloss. Der Antikörper verlängerte das
\end{abstract}

Gesamtüberleben (primärer Endpunkt) um $35 \%$ von 7,4 Monaten auf 10,1 Monate und das progressionsfreie Überleben um 2,3 Monate (5,6 Monate versus 3,3 Monate). Mit Ausnahme von Infusions- und Hautreaktion zeigte sich keine Zunahme der Toxizität. Auftreten und Schwere der akneiformen Hautreaktion korrelierten mit dem Überleben. Daten zur Lebensqualität der Patienten zeigten ebenfalls einen Vorteil: Schmerzen sowie Beschwerden beim Schlucken, Sprechen und Essen verbesserten sich im Prüfarm deutlich. Alle Subgruppen profitierten gleichermaßen von der Behandlung, so Hildebrandt.

Die European Society for Medical Oncology (ESMO) empfiehlt in ihren aktuellen Leitlinien zur Therapie von Kopf-Hals-Tumoren (2009) Cetuximab kombiniert mit platinbasierter Chemotherapie in der Erstlinienbehandlung rezidivierter und/oder metastasierter SCCHN mit Evidenzgrad A. Auch bei lokal fortgeschrittenen Tumoren gilt für Cetuximab in Kombination mit Strahlentherapie der höchste Evidenzgrad. koc

Satelliten-Symposium „Die Therapie von Kopf-Hals-Tumoren: Eine interdisziplinäre Herausforderung" im Rahmen der 16. Jahrestagung der Deutschen Gesellschaft für Radioonkologie, Magdeburg, 4. Juni 2010. Veranstalter: Merck Serono

\section{Durchblick für HNO-Chirurgen}

— Vielseitigkeit, funktionell durchdachtes Design und ergonomische Bedienung kennzeichnen das Leica Mikroskop M32O F12, ein kompaktes Instrument für HNOChirurgen bei Routine-Eingriffen. Zu den wichtigsten funktionalen Merkmalen des Geräts gehören intern verlegte Kabel, eine leicht zu reinigende, antimikrobiell beschichtete Oberfläche und ein einfacher zuverlässiger Betrieb.

Die Leica-Optik ist kombiniert mit modernster LED-Beleuchtung und liefert klare helle und absolut naturgetreue Bilder von großer Tiefenschärfe. Die LEDs verfügen über eine Lebensdauer von 60.000 Stun- den, was im Vergleich zu konventionellen Beleuchtungssystemen die Betriebskosten spürbar senkt.

Das Mikroskop kann zusätzlich mit HDBildtechnik ausgestattet werden. Dies ermöglicht nicht nur die Aufnahme von Fotos und Videos, deren Darstellung auf einem HD-Monitor und die Speicherung auf einer SD Memory Card, sondern ermöglicht auch neue Optionen zur Dokumentation, Präsentation und Beratung.

gz

Nach Informationen von Leica Mikrosystems 